BNL-108195-2015-JA

\title{
Production of Long Chain Alcohols and Alkanes Upon Coexpression of an Acyl-ACP Reductase and Aldehyde-Deformylating Oxgenase with a Bacterial Type-I Fatty Acid Synthase in E. coli
}

\author{
Dan Coursolle, ${ }^{a}$ Jiazhang Lian, ${ }^{\text {J John Shanklin, }}$ \\ and Huimin Zhao ${ }^{\text {ac }}$ \\ ${ }^{a}$ Department of Chemical and Biomolecular Engineering, \\ Institute for Genomic Biology, University of Illinois \\ at Urbana-Champaign, Urbana, IL 61801 \\ b Brookhaven National Laboratory, P.O. Box 5000, \\ 50 Bell Avenue, Building 463, Upton, NY 11973 \\ c Departments of Chemistry, Biochemistry and Bioengineering, \\ University of Illinois at Urbana-Champaign, Urbana, IL 61801
}

Accepted to Molecular Biosystems

June 2015

Biological, Environmental \& Climate Sciences Department Brookhaven National Laboratory

U.S. Department of Energy Office of Science Office of Basic Energy Sciences

Notice: This manuscript has been authored by employees of Brookhaven Science Associates, LLC under Contract No. DE-SC0012704 with the U.S. Department of Energy. The publisher by accepting the manuscript for publication acknowledges that the United States Government retains a non-exclusive, paid-up, irrevocable, world-wide license to publish or reproduce the published form of this manuscript, or allow others to do so, for United States Government purposes. 


\section{DISCLAIMER}

This report was prepared as an account of work sponsored by an agency of the United States Government. Neither the United States Government nor any agency thereof, nor any of their employees, nor any of their contractors, subcontractors, or their employees, makes any warranty, express or implied, or assumes any legal liability or responsibility for the accuracy, completeness, or any third party's use or the results of such use of any information, apparatus, product, or process disclosed, or represents that its use would not infringe privately owned rights. Reference herein to any specific commercial product, process, or service by trade name, trademark, manufacturer, or otherwise, does not necessarily constitute or imply its endorsement, recommendation, or favoring by the United States Government or any agency thereof or its contractors or subcontractors. The views and opinions of authors expressed herein do not necessarily state or reflect those of the United States Government or any agency thereof. 


\title{
Production of Long Chain Alcohols and Alkanes Upon Coexpression of an Acyl-ACP Reductase and Aldehyde-Deformylating Oxygenase with a Bacterial Type-I Fatty Acid Synthase in E. coli $†$
}

\author{
Dan Coursolle, ${ }^{a}$ Jiazhang Lian, ${ }^{a}$ John Shanklin, ${ }^{b}$ and Huimin Zhao ${ }^{a c}$
}

\begin{abstract}
Microbial long chain alcohols and alkanes are renewable biofuels that could one day replace petroleum-derived fuels. Here we report a novel pathway for high efficiency production of these products in Escherichia coli strain BL21(DE3). We first identified the acyl-ACP reductase/aldehyde deformylase combinations with the highest activity in this strain. Next, we used catalase coexpression to remove toxic byproducts and increase the overall titer. Finally, by introducing the type-I fatty acid synthase from Corynebacterium ammoniagenes, we were able to bypass host regulatory mechanisms of fatty acid synthesis that have thus far hampered efforts to optimize the yield of acyl-ACP-derived products in BL21(DE3). When all these engineering strategies were combined with subsequent optimization of fermentation conditions, we were able to achieve a final titer around $100 \mathrm{mg} / \mathrm{L}$ long chain alcohol/alkane products including a $57 \mathrm{mg} / \mathrm{L}$ titer of pentadecane, the highest titer reported in E. coli BL21(DE3) to date. The expression of prokaryotic type-I fatty acid synthases offer a unique strategy to produce fatty acid-derived products in E. coli that does not rely exclusively on the endogenous type-II fatty acid synthase system.
\end{abstract}

\section{Introduction}

The depletion of fossil fuels and dependence on imported oils has caused a high demand for generating energy from sustainable sources for energy security. Strategies to transition dependence on imported petrochemicals have focused both on developing domestic fossil fuel resources and the production of renewable fuels compatible with existing infrastructure. ${ }^{1-3}$ Promising “drop-in" biofuels include iso-butanol, fatty acid ethyl esters (FAEE, bio-diesel), and long chain alkanes, all of which can be produced in Escherichia coli upon the introduction of heterologous biosynthetic pathways. Among these renewable fuels, alkanes are particularly appealing as a transportation fuel replacement because their structural similarity with gasoline allows for rapid integration into existing fuel refining, transportation, and distribution infrastructure. Long chain alkanes are naturally produced by some cyanobacteria and the enzymes responsible for their production can be expressed in $E$. coli where they convert endogenous metabolites to long chain alkanes, alkenes, and alcohols. $^{4}$

Fatty acid synthesis (FAS) in E. coIi involves elongation of acyl-acyl carrier protein (ACP) precursors using activated acetyl-CoA units (malonyl-ACP) and subsequent reduction, dehydration, and reduction reactions to form fatty acyl-ACPs (Figure 1). E. coli has a native type-II FAS system, where the enzymes performing the chemical transformations exist as distinct proteins, as opposed to type-I FAS where the reactions are performed by large multi-subunit polypeptides. ${ }^{5,} 6$ Unfortunately, due to relatively low flux and tight regulation of its endogenous FAS pathway, wild-type $E$. coli is not capable of producing high titers of fatty acids, i.e., less than $20 \mathrm{mg} / \mathrm{L}$ in a batch culture for strain $\mathrm{DH} 1,{ }^{7} 800 \mathrm{mg} / \mathrm{L}$ in strain $\mathrm{MG} 1655^{8}$ or $130 \mathrm{mg} / \mathrm{L}$ in a fed-batch culture for BL21(DE3). ${ }^{9}$ Flux through the FAS pathways can be increased by overexpressing thioesterases, acetyl-CoA carboxylases, and global regulators, $8,10-12$ and sugar conversion to fatty acid rates as high as $73 \%$ of the theoretical limit, at titers of $5 \mathrm{~g} / \mathrm{L}$, have been achieved. ${ }^{8}$ Recently higher titer $(8.6 \mathrm{~g} / \mathrm{L})$ was achieved by modular optimization of the multi-gene pathways for fatty acid production followed by fed-batch fermentation. ${ }^{10}$ Despite the successful over-production of fatty acids, progress towards optimizing their conversion to biofuels has advanced more slowly. To date, the highest production of alkanes reported was around $800 \mathrm{mg} / \mathrm{L}$ using fed-batch fermentation, ${ }^{11}$ and the highest reported de novo FAEE production is $1.5 \mathrm{~g} / \mathrm{L}$ using $2 \%$ glucose, a $28.0 \%$ theoretical yield. ${ }^{12}$ Recently, de novo production of fatty alcohol was also attempted in E. coli, with the highest titer over $1.6 \mathrm{~g} / \mathrm{L}$ and yield over $0.13 \mathrm{~g} / \mathrm{g}$ carbon source achieved. ${ }^{13}$ Possible reasons for the difference in the titers of free fatty acids (FFAs) and downstream biofuels range from toxicity of products or intermediates in the biosynthesis of biofuels to poor specificity, turnover, or expression of the 


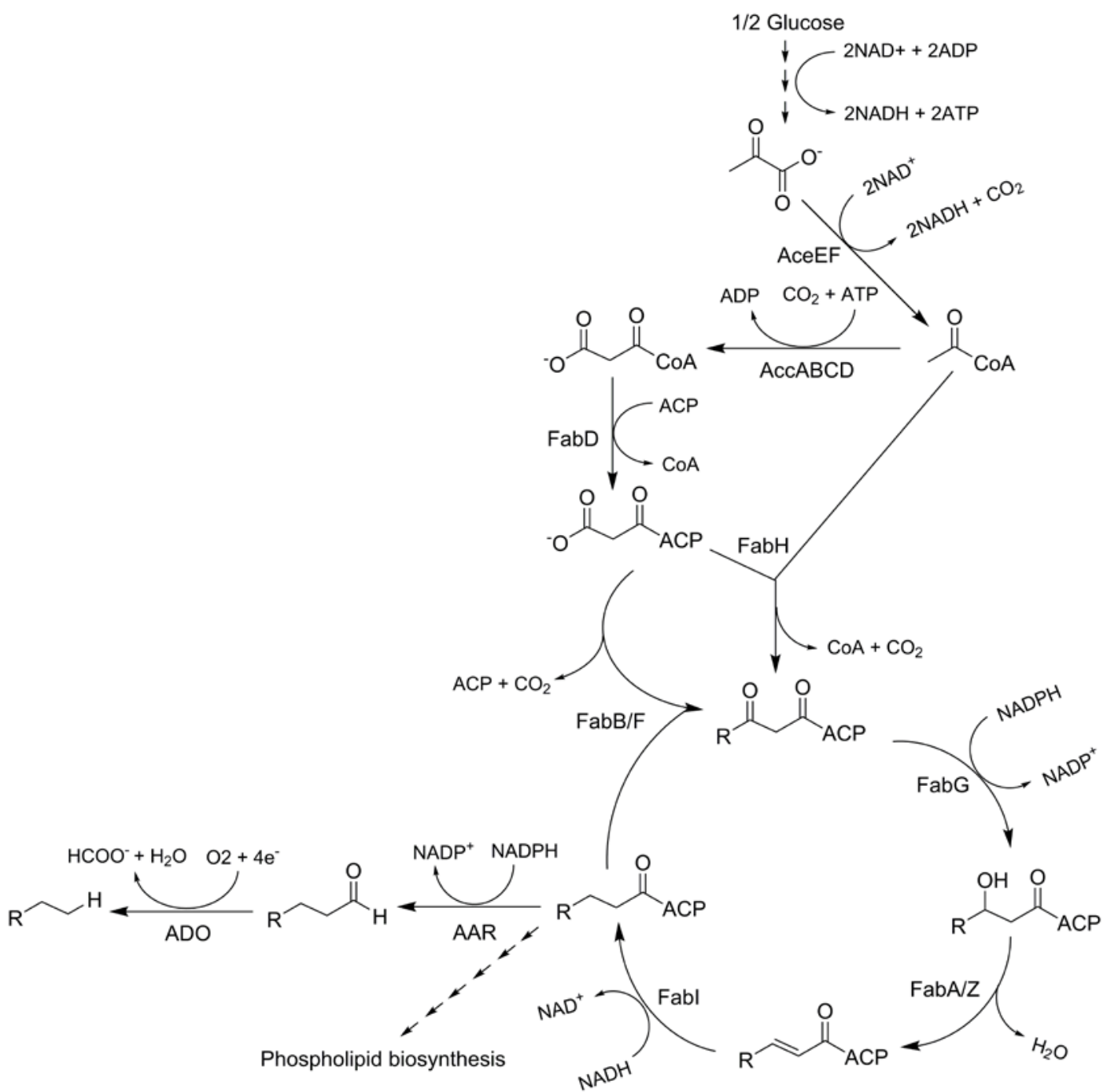

Figure 1. Type-II fatty acid and long chain alkane biosynthetic pathway using heterologous genes in E. coli. Abbreviations: ATP Adenosine triphosphate, ADP - Adenosine diphosphate, $\mathrm{CO}_{2}$ - carbon dioxide, $\mathrm{O}_{2}$ - oxygen, CoA - Coenzyme A, ACP - acyl carrier protein, $\mathrm{NADP}^{+}$- nicotinamide adenine dinucleotide phosphate, $\mathrm{NAD}^{+}$- Nicotinamide adenine dinucleotide, $\mathrm{HCOO}^{-}$formate, $4 \mathrm{e}^{-}$- four electrons transferred via an unknown mechanism. Enzyme abbreviations: AceEF - pyruvate dehydrogenase, AccABCD - acetyl-CoA carboxylase, FabD - malonyl-CoA:ACP S-malonyltransferase, FabH - $\beta$-ketoacyl-ACP synthase, FabG $\beta$-ketoacyl-ACP reductase, FabA/Z - $\beta$-ketoacyl-ACP dehydratase, FabI - enoyl-ACP reductase, FabB/F - $\beta$-ketoacyl-ACP synthase, AAR - acyl-ACP reductase, ADO - aldehyde deformylating oxygenase.

catalytic enzymes themselves. An alternative explanation is that alkane/biodiesel producing enzymes that require FFAs to be activated with ACP or CoA, while overexpression of thioesterases and regulators may increase FFA concentration., 13 Moreover, since FFAs are secreted from cells in overproducing strains, ${ }^{9}$ the intracellular concentration of alkane/biodiesel precursors may be insufficient to achieve high flux. Regardless, fatty acid-derived biofuels remains a growing field with several private and public companies attempting to take production to a sustainable commercial level.

The acyl-ACP reductase (AAR) of Synechococcus elongatus and aldehyde decarbonylase, later renamed aldehydedeformylating oxygenase (ADO) based on mechanistic studies, ${ }^{14-16}$ are sufficient for alkane/alkene production in $E$. coli. ${ }^{4}$ Since a reducing system is required for ADO activity, the electron transfer system should be included together with the in vitro reconstituted ADO for alkane production from fatty aldehyde. $^{17}$ Alternatively, self-sufficient ADO could be engineered by fusing ADO with an electron transfer system. ${ }^{18} \mathrm{It}$ was also reported that the production of alkanes could be increased by spatial organization of AAR and ADO. ${ }^{19}$ We analyzed 7 AAR homologs from different cyanobacterial species for the highest accumulation of fatty aldehydes/alcohols, and found that the previously reported $S$. elongatus PCC7942 reductase has the highest in vivo activity in E. coli BL21(DE3) for producing long chain alcohols, where the other homologs showed $>10$ fold less activity. We next evaluated 8 ADOs from cyanobacteria and discovered a homolog from Cyanothece sp. pcc 7425 that when expressed with AAR results in a $\sim 5 \mathrm{x}$ higher accumulation of long chain alcohols and alkanes in E. coli BL21(DE3) for a combined titer of $\sim 10 \mathrm{mg} / \mathrm{L}$ pentadecane, heptadecane, tetradecanol, and hexadecanol (PHTH).

While acyl-ACPs inhibit several enzymes in type-II FAS pathways, $^{20,21}$ they may not inhibit type-I FAS enzymes where the reaction center is contained in a single multi-domain 
polypeptide, $^{5}$ although acyl-CoAs then may become the potential repressors. ${ }^{22}$ Most recently, when this manuscript was under review, an orthogonal FAS system was developed by heterologous expression of type-I FAS for oleochemical production. Among several type I FAS enzymes tested, FAS1A from Corynebacterium glutamicum (cgFAS1A) was found to be the most active in E. coli. ${ }^{23}$ Compared with the control strain without any engineering of acyl-CoA overproduction, the production of methyl ketones was improved from less than 5 $\mathrm{mg} / \mathrm{L}$ to about $28 \mathrm{mg} / \mathrm{L}$. Interestingly, the same engineering strategy resulted in no improvement in the production of fatty alcohols. We hypothesized that expression of FAS-B, a type-I FAS from Corynebacterium ammoniagenes, ${ }^{24,} 25$ with alkane biosynthetic genes might bypass the feedback inhibition of acyl-ACPs on type-II FAS in E. coli and improve PHTH titers. In the present study, overexpression of FAS-B increased the yield of palmitic acid (when expressed alone) and PHTH (when expressed with AAR and/or ADO) in E. coli strain BL21(DE3). The highest alkane/alcohol producing strain we obtained could make $70.5 \mathrm{mg} / \mathrm{L} \mathrm{PHTH}$, or $2 \%$ of the theoretical maximum yield. Based on our results using a type-I FAS, we present here a model for the biosynthesis of PHTH products in E. coli that involves an equilibrium with acyl-CoAs, FFAs, and acyl-ACPs using modules from actinobacteria, enterobacteria, and cyanobacteria. Using this model we suggest potential routes to redirect flux to increase the titer of PHTH-like products.

\section{Results}

\section{In vivo AAR activity}

Our first objective was to identify a high-producing alkane biosynthesis pathway in E. coli BL21(DE3). The first step in the cyanobacterial production of fatty acids is the conversion of long chain acyl-ACPs to aldehydes catalyzed by AAR. The AAR from $S$. elongatus is sufficient for long chain aldehyde synthesis in E. coli MG1655. ${ }^{4}$ The sequence for $S$. elongatus PCC7942 AAR was compared to the genomes of 6 different cyanobacterial strains generously proved by Himadri Pakrasi (Table 1). All but one of these strains contained an AAR homolog, which was cloned into a pACYCduet-based expression vector and transformed into BL21(DE3) and subsequent strains assayed for in vivo long chain aldehyde and alcohol production. While long chain aldehydes were not detected in any samples due to native alcohol dehydrogenase activity, ${ }^{4}$ several strains produced long chain alcohols, the most prominent of which was hexadecanol. The best yielding strain contained the AAR from $S$. elongatus (BL21:AAR), which made $8.15 \mathrm{mg} / \mathrm{L}$ long chain alcohols (LCA). Since this AAR demonstrated the highest in vivo activity, it was selected for subsequent experiments.

Table 1. Production of tetra- and hexadecanol by E. coli expressing AAR homologs

\begin{tabular}{llll}
\hline Origin & Locus tag & \% identity & LCA (mg/L) \\
\hline Synechococcus elongatus PCC 7942 & SynpcC7942_1594 & 100 & $8.15 \pm .078$ \\
Cyanothece sp. Strain ATCC 51142 & cCe_1430 & 68 & $0.326 \pm .071$ \\
Cyanothece sp. PCC 7822 & - & N/A & N/A \\
Synechocystis sp. PCC 6803 & SYNPCCP_2249 & 69 & $0.385 \pm .078$ \\
Cyanothece sp. PCC 8801 & PCC8801_0872 & 69 & $0.239 \pm .028$ \\
Cyanothece sp. PCC 8802 & Cyan8802_0898 & 69 & $0.359 \pm .021$ \\
Cyanothece sp. PCC 7425 & Cyan7425_0399 & 70 & $0.206 \pm .036$ \\
\hline
\end{tabular}

Abbreviations: LCA - long chain alcohols, N/A - not applicable

Error shown is one standard deviation from biological triplicates

Table 2. Production of alkanes by E. coli expressing ADO homologs and S. elongatus AAR

\begin{tabular}{llll}
\hline Origin & Locus tag & \% identity & Alkane (mg/L) \\
\hline Synechococcus elongatus PCC 7942 & Synpcc7942_1593 & 100 & $1.48 \pm 0.49$ \\
Cyanothece sp. Strain ATCC 51142 & †cce_0778 & 73 & $2.56 \pm 0.47$ \\
Cyanothece sp. PCC 7822 & - & N/A & N/A \\
Synechocystis sp. PCC 6803 & †SYNPCCP_2250 & 72 & $0.85 \pm 0.12$ \\
Cyanothece sp. PCC 8801 & PCC8801_0455 & 71 & $0.56 \pm 0.62$ \\
Cyanothece sp. PCC 8802 & Cyan8802_0468 & 71 & ND \\
Cyanothece sp. PCC 7425 & †Cyan7425_2986 & 59 & $1.75 \pm 0.48$ \\
& †Cyan7425_0398 & 71 & $7.75 \pm 0.93$ \\
\hline
\end{tabular}

Abbreviations: N/A - not applicable, ND - none detected

Alkane concentration corresponds to the addition of pentadecane and heptadecane

Error shown is one standard deviation from biological triplicates

$\ddagger$ - Assayed in, ${ }^{4}$ but no yields given.

\section{In vivo ADO activity}

The second step in cyanobacterial alkane production is the oxidative deformylation of long chain aldehydes to alkanes and is catalyzed by ADO. ${ }^{16}$ Using the AAR from $S$. elongatus PCC 7942, we added ADO homologs from the previously identified cyanobacterial strains into the pACYCduet-derived vector containing $S$. elongatus AAR. As shown in Table 2, several of these ADOs were able to produce alkanes when co-expressed with S. elongatus PCC 7942 AAR. The ADO demonstrating the highest in vivo activity was from Cyanothece sp. PCC 7425 locus tag Cyan7425_0398 (BL21:AAR/ADO2). This strain was able to produce $7.8 \mathrm{mg} / \mathrm{L}$ alkanes (mostly pentadecane), or 
around 5 times more than the ADO from S. elongatus (ADO1) under these conditions. Cyan7425_0398 was previously stated to have less activity than that of $S$. elongatus in E. coli MG1655, ${ }^{4}$ however absolute titers were not given to make an accurate comparison. BL21:AAR/ADO1 (containing the ADO from $S$. elongatus) was constructed as a reference and made $1.48 \mathrm{mg} / \mathrm{L}$ long chain alkane products (Table 2).

\section{Catalase coexpression increases alkane yields}

In previous work, it was reported that catalase, when added exogenously or fused with ADO, can increase deformylation activity by removing inhibitory peroxide molecules. ${ }^{26}$ Therefore, we attempted to modify our engineered alkane biosynthetic pathway to increase alkane yields by coexpressing a catalase. There are two catalase homologs present in the BL21(DE3) genome at loci B21_03777 (HPI) and B21_01689 (HPII), respectively. These two catalases were cloned into a pRSFduet-derived vector and transformed into BL21:AAR/ADO1 and BL21:AAR/ADO2 to make BL21:AAR/ADO1/HPI, BL21:AAR/ADO1/HPII, BL21:AAR/ADO2/HPI, and BL21:AAR/ADO2/HPII. While HPI did not have a noticeable effect on alkane yields, coexpression of HPII was able to increase the alkane titer when expressed with each ADO homolog (Figure 2). The highest PHTH titer was achieved by BL21:AAR/ADO2/HPII which was $12.3 \mathrm{mg} / \mathrm{L}$, with the major product remaining pentadecane.

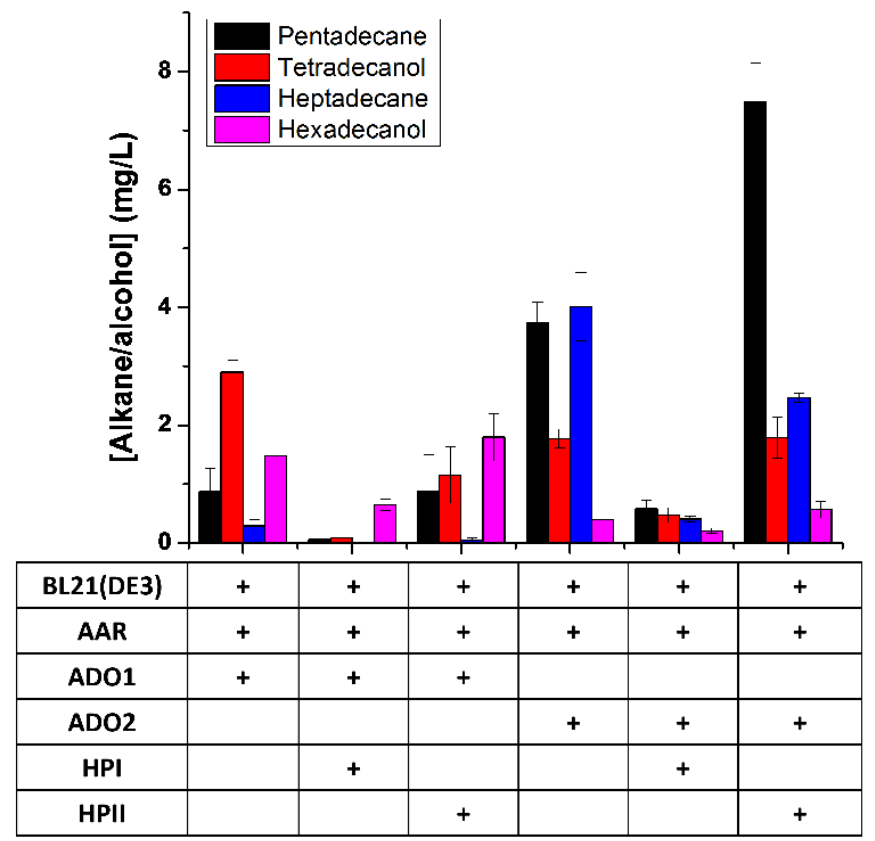

Figure 2. Long chain alcohol and alkane production by recombinant strains expressing catalase. Error shown is one standard deviation from biological triplicates.

\section{In vivo FAS-B activity}

FAS-B from C. ammoniagenes is a type-I FAS that synthesizes fatty acids (mostly palmitic acid) from acetyl-CoA precursors. $^{24,} 25$ Interestingly, it was found later that FAS-B was able to use acyl-CoAs with different chain lengths as the primer units for fatty acid biosynthesis. ${ }^{27}, 28$ We wanted to see if coexpression of a bacterial type-I FAS would increase the flux through heterologous alkane pathways. The plasmid pGM44, which contains the FAS-B and pptA (phosphopantetheine: protein transferase necessary for FAS-B activation by phosphopantetheinylation) genes from $C$. ammoniagenes, was transformed into BL21(DE3) to make BL21:FAS-B. Next, the strain BL21:FAS-B was assayed for fatty acid production levels and distributions and compared to BL21(DE3). Wild-type BL21(DE3) mainly produces myristic, palmitic, palmitoleic, and oleic acids. ${ }^{29}$ As shown in Figure 3, BL21:FAS-B produced around 2.5 fold more palmitic acid than wild-type BL21(DE3), suggesting that the imported FAS-B was properly expressed and that it was activated by PptA. There was also an increase in palmitoleic acid, though the reasons for this are less obvious. We did not observe a significant change in myristic or oleic acid produced during these experiments. Since the total fatty acid titer was increased upon its expression, FAS-B is likely not subject to feedback inhibition by acyl-ACPs.

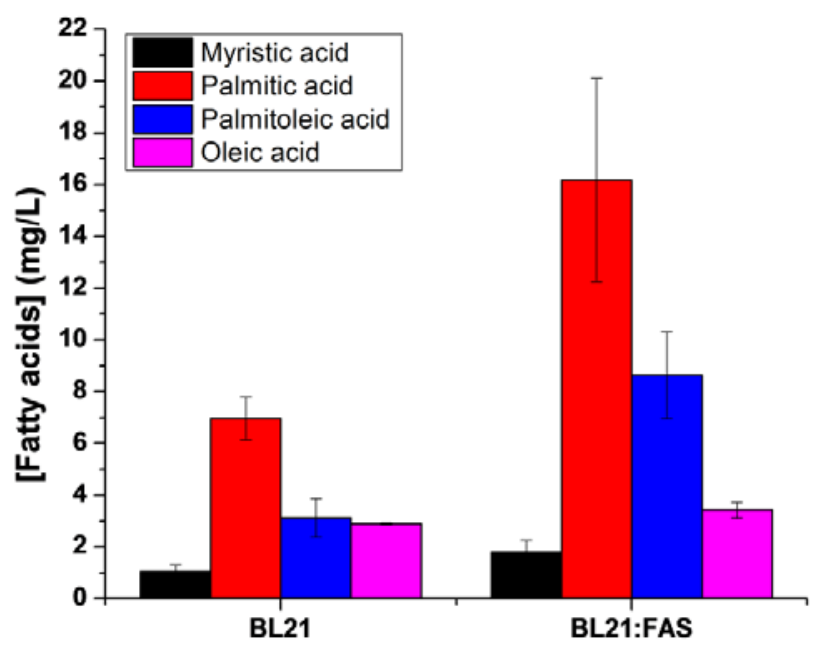

Figure 3. Fatty acid production in BL21(DE3) strains expressing $F A S-B$. Error shown is one standard deviation from biological triplicates.

\section{Coexpression of a type-I FAS with AAR and ADO}

Since an active alkane biosynthetic pathway was identified and in vivo FFA production was observed that bypasses feedback inhibition native to $E$. coli FAS, FAS-B was moved into BL21:AAR/ADO1 and BL21:AAR/ADO2 to create BL21:AAR/ADO1/FAS and BL21:AAR/ADO2/FAS. Coexpression of FAS-B with both alkane biosynthetic pathways increased PHTH titers, most dramatically in the strain expressing ADO1 (Figure 4). The largest increase was observed in pentadecane and hexadecanol, an expected result as both of these products are derived from palmitic acid, the main product synthesized by FAS-B. The total yield of PHTH in our highest producing strain, BL21:AAR/ADO1/FAS, was $70.5 \mathrm{mg} / \mathrm{L}$. Interestingly, ADO2 performed better when co-expressed with AAR alone, while alkane production was much lower that of ADO1 when FAS-B was also included, which might result from the poor growth of BL21:AAR/ADO2/FAS. Next, HPII was added to BL21:AAR/ADO1/FAS and BL21:AAR/ADO2/FAS to make BL21:AAR/ADO1/FAS/HPII and BL21:AAR/ADO2/FAS/HPII to test if peroxide removal would help increase PHTH yields. Surprisingly, the addition of catalase actually caused a dramatic decrease in the titer of PHTH products (data not shown), probably due to the metabolic burden and/or competition of several IPTG-inducible expression of heterologous proteins. Although the cell growth of BL21:AAR/ADO1/FAS and BL21:AAR/ADO1/FAS/HPII was similar (Figure S1, ESI†), significant difference in the 
expression of heterologous proteins was observed. As shown in Figure S2 (ESI $\dagger$ ), the expression level of both alkane producing enzymes, AAR and ADO1, was much lower in the HPII coexpressing strain.

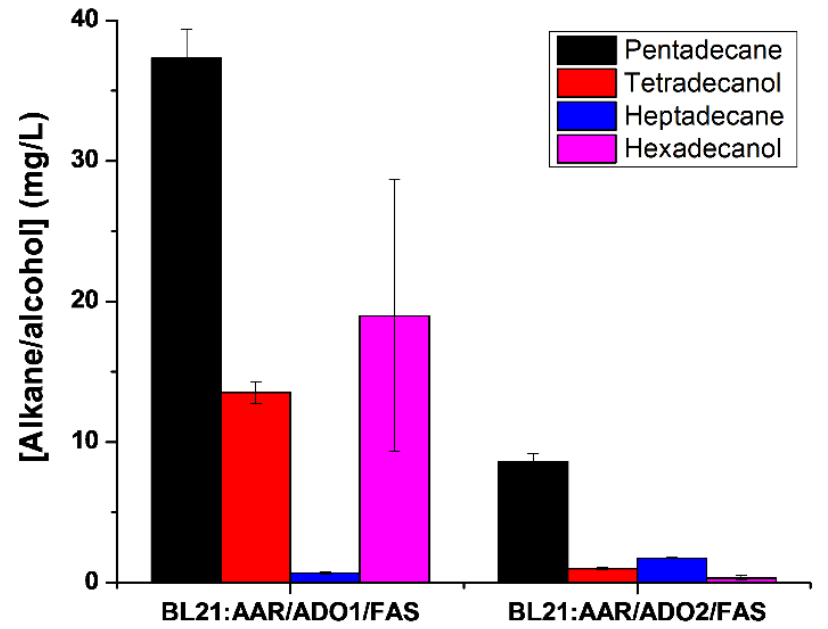

Figure 4. Long chain alcohol and alkane production by recombinant strains expressing $F A S-B$. Error shown is one standard deviation from biological triplicates.

\section{Optimization of alkane production with BL21:AAR/ADO1/FAS}

Since all heterologous genes were put under the control of IPTG-inducible promoters, the timing of IPTG addition and the concentration of the inducer might have a significant impact on the expression of heterologous genes and thus the production of alkanes. To study the effect of induction timing on alkane production, IPTG was added at early-log $\left(\mathrm{OD}_{600} \sim 0.5\right)$, mid-log $\left(\mathrm{OD}_{600} \sim 1.0\right)$, late-log $\left(\mathrm{OD}_{600} \sim 2.0\right)$, and stationary phase $\left(\mathrm{OD}_{600} \sim 3.0\right)$, respectively. As shown in Figure 5A, induction at high cell density led to significant lower production of long chain alcohols and alkanes, probably due to the low activity of cells at late growth stage. Next we investigated the effect of IPTG concentration on the biosynthesis of alkanes. Figure 5B indicated that higher inducer concentration resulted in higher long chain alcohol and alkane production, with the highest level of PHTH around $100 \mathrm{mg} / \mathrm{L}$ achieved with $0.75 \mathrm{mM}$ IPTG.

\section{Discussion}

In this study, we demonstrate the ability of E. coli BL21(DE3) to synthesize pentadecane, hexadecanol, heptadecane, and tetradecanol at increased titers upon the expression of catalase and a type-I FAS in conjunction with optimized AAR/ADO combinations. In previous studies, ${ }^{4}$ higher titers (200-300 $\mathrm{mg} / \mathrm{L}$ ) were reported using the $E$. coli strain MG1655. Another study aimed to expand the alkane profiles using BL21(DE3) resulted in the production of total alkanes at a titer of $40 \mathrm{mg} / \mathrm{L}$ when AAR/ADO was expressed, ${ }^{30}$ which was quite similar to what was achieved in this study. This raises the interesting question of why two $E$. coli strains would demonstrate such dramatic differences in alkane accumulation when expressing the same AAR/ADO and cultured under similar conditions. The most likely answer involves the cytoplasmic availability of the AAR substrate acyl-ACPs. A higher concentration of acylACPs in MG1655 could be the driving force causing higher flux through the heterologous alkane biosynthetic pathway. Support for this hypothesis comes from studies showing other acyl-ACP utilizing pathways show relatively low activity in
BL21(DE3) when compared to MG1655. ${ }^{29}$, 31, 32 While intracellular acyl-ACP concentrations were not determined in these studies, a reasonable explanation for differential activity of similar pathway activities in non-identical strains would be the intracellular concentration of this shared precursor. The low acyl-ACP hypothesis could also explain the lack of detectable levels of long chain aldehyde products in BL21(DE3) in that an insufficient concentration of precursor may reduce the overall activity of AAR which enables the ADO and alcohol dehydrogenases to scavenge nearly all aldehyde products. In other genetic backgrounds with higher levels of acyl-ACPs, there would be an increased flux through AAR resulting in aldehydes accumulation at a faster rate than ADO can catalyze their oxidative deformylation.

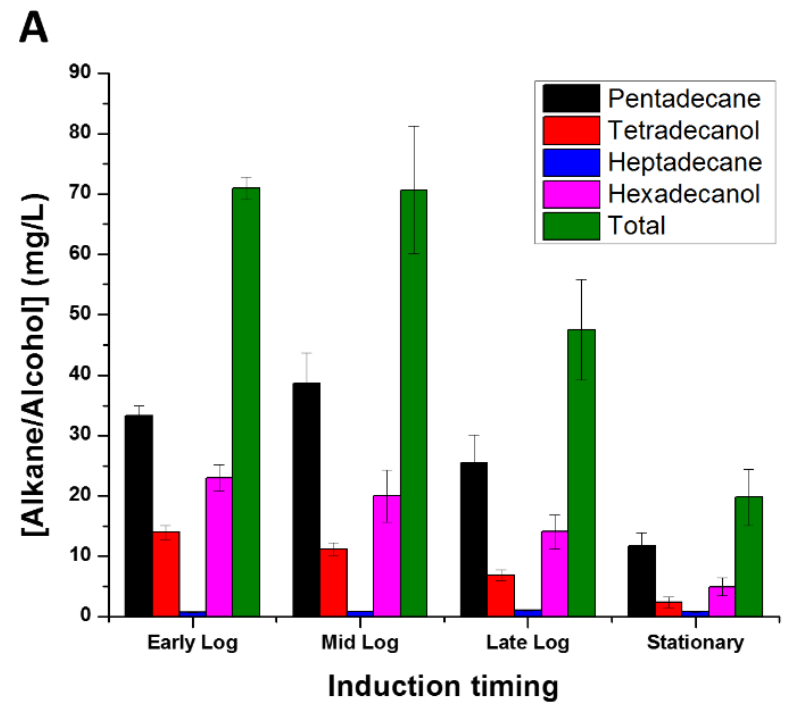

B

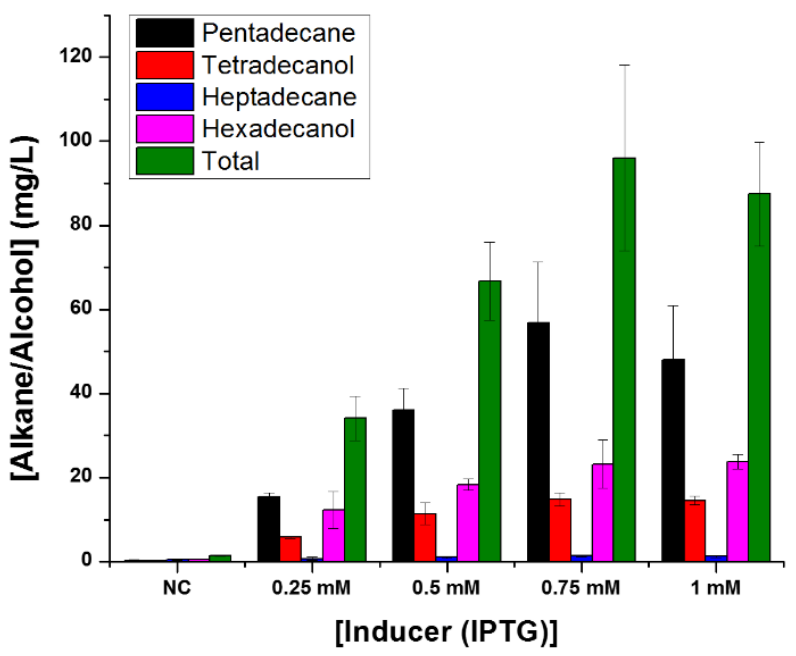

Figure 5. Optimization of fermentation conditions for long chain alcohol and alkane production. BL21:AAR/ADO1/FAS was induced by IPTG at different growth stage (early-log, midlog, late-log, and stationary phase, respectively) or with different concentration $(0,0.25,0.5,0.75$, and $1.0 \mathrm{mM}$, respectively).

The mechanism of interaction between FAS-B and AAR remains unknown. It is possible that the acyl-CoAs released by 
actinobacterial type-I FASs ${ }^{5,33-35}$ can directly react with AAR to make long chain aldehydes. However, the affinity of AAR for Acyl-CoAs is 16-fold lower than for acyl-ACPs making this explanation unlikely. ${ }^{4}$ Another possibility can be visualized in Figure 6, where acyl-CoAs released by FAS-B are in equilibrium with FFAs and acyl-ACPs using native E. coli enzymes and the alkane module using heterologous cyanobacterial enzymes. In this model, since FAS-B may not be controlled by the same feedback inhibition as the native type-II FAS system, local concentrations of acyl-ACPs would be increased by the higher levels of acyl-CoAs and FFAs, increasing the final alkane titer. It is also possible that the intracellular acetyl- and malonyl-CoA concentrations are maintained at higher levels than their ACP counterparts, driving the flux forward when an acyl-CoA utilizing FAS such as FAS$\mathrm{B}$ is present. Indeed, overexpression of acyl-CoA-utilizing fatty acid degradation pathways has been demonstrated to redirect carbon flux towards fatty acid biosynthesis, likely due to relatively high levels of intracellular acyl-CoAs to acylACPs. ${ }^{36,37}$ In this case, it seems increasing extracellular FFA concentration by either exogenous addition or endogenous overproduction should also drive the flux forward. This may not be the case, however, because the intracellular concentrations of FFAs and acyl-CoAs in these cases are not known.

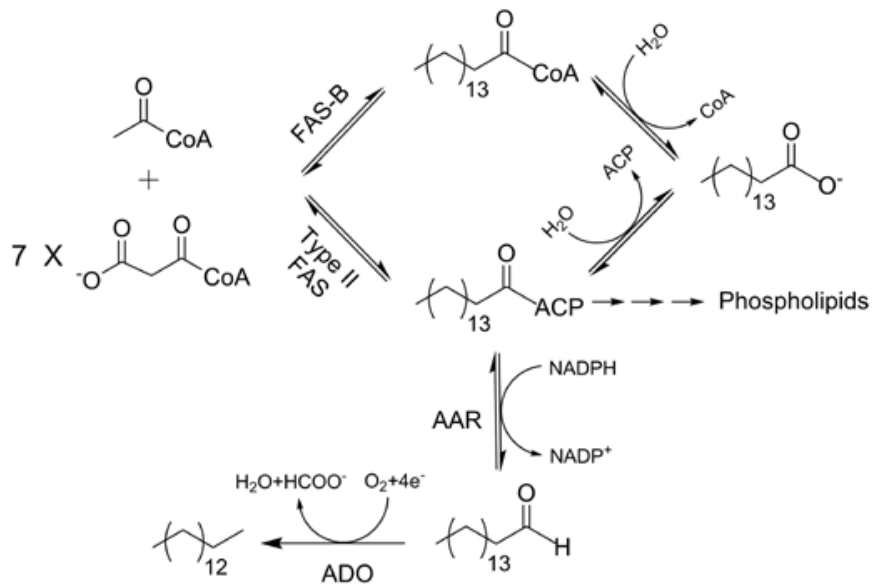

Figure 6. Model for pentadecane production in E. coli strain BL21(DE3). This model depicts the complex equilibrium between acetyl-CoA, malonyl-CoA, palmitoyl-CoA, palmitic acid, palmitoyl-ACP, and pentadecane even though the reactions may be catalyzed by different enzymes. Changes in the driving force can either push or pull flux towards the desired product, pentadecane.

We observed an increase in both palmitic and palmitoleic acid upon overexpression of FAS-B in E. coli BL21(DE3). Corynebacterium ammoniagenes produced both saturated and unsaturated (including C16:1) fatty acids, due to the presence of two functional type-I FAS, FAS-A and FAS-B. In the FAS-A knock-out strain, no unsaturated fatty acids were detected, indicating that FAS-B can only make saturated fatty acids. ${ }^{35}$ This result was further confirmed by in vitro assays. ${ }^{25}$ As the major product of FAS-B, ${ }^{19,20}$ one would expect palmitic acid to build up in these engineered strains, but the increase in palmitoleic acid is more difficult to explain. In many prokaryotes including $E$. coli, fatty acid desaturation proceeds using an anaerobic mechanism that isomerizes trans-2decanoly-ACP to cis-3-decanoyl-ACP using the FabA protein. ${ }^{26,27}$ Since no C10 carbon products are released from
FAS-B, it is difficult to understand how increasing palmitic acid concentrations would affect unsaturated fatty acid synthesis. One possible explanation involves the feedback inhibition of acyl-ACPs in equilibrium with acyl-CoAs (Figure 6). Increased acyl-ACP concentrations will retard type-II FAS, perhaps increasing the local concentration of trans-2-decanoylACP, which could divert a larger fraction of fatty acids to the unsaturated pathway.

\section{Experimental}

\section{Strains and vectors}

NEB 5-alpha chemically competent E. coli (New England BioLabs, Ipswich, MA) were used for plasmid amplification, construction, and verification. E. coli strains were routinely cultivated in lysogeny broth (LB) or M9 minimal media with $3 \%$ glucose, $2 \mathrm{mM}$ magnesium sulphate, $100 \mu \mathrm{M}$ calcium chloride, and a 1:1000 dilution of a mineral mix containing 27 $\mathrm{g} / \mathrm{L} \mathrm{FeCl}_{3} \bullet 6 \mathrm{H}_{2} \mathrm{O}, 2 \mathrm{~g} / \mathrm{L} \mathrm{ZnCl}_{2} \bullet 4 \mathrm{H}_{2} \mathrm{O}, 1.9 \mathrm{~g} / \mathrm{L} \mathrm{CuSO}_{4} \bullet 5 \mathrm{H}_{2} \mathrm{O}, 0.5$ $\mathrm{g} / \mathrm{L} \mathrm{H}_{3} \mathrm{BO}_{3}, 1 \mathrm{~g} / \mathrm{L}$ Thiamine as indicated. All E. coli strains were cultured at $37^{\circ} \mathrm{C}$, including seed culture in LB media, preinduction culture in M9 media, and post-induction culture for alkane biosynthesis. When cultures were not to be extracted, $E$. coli strains were grown in $14 \mathrm{~mL}$ polypropylene round bottom tubes (Thermo Fisher, Pittsburgh, PA) with shaking at 250 RPM on a New Brunswick Scientific (Eppendorf, Hamburg, Germany) I24 incubator shaker. Chloramphenicol, ampicillin, and kanamycin were added at 50, 100, and $50 \mu \mathrm{g} / \mathrm{mL}$ respectively, when necessary. pRSFduet, and pACYCduet were obtained from Novagen (Merck, Darmstadt, Germany). Plasmid pGM44 was obtained from Eckhart Schweizer. ${ }^{19,20}$

\section{DNA manipulation}

Genomic DNA for cyanobacterial strains was generously donated by Himadri Pakrasi. Primers used for gene amplification are denoted in Table S1 (ESI†). Plasmids were isolated using a QIAprep Spin Miniprep Kit (Qiagen, Valencia, CA) according to the manufacturer's protocol. All enzymes for recombinant DNA cloning were obtained from New England BioLabs unless indicated otherwise. After digestion, ligation, and PCR, DNA was purified using QIAquick PCR Purification or Gel Extraction Kits. All plasmids and strains used in this study are summarized in Table 3.

\section{Fatty acid analysis}

E. coli strains were grown in $5 \mathrm{~mL}$ modified M9 minimal media in glass culture tubes and were induced with $0.5 \mathrm{mM}$ isopropyl $\beta$-D-1thiogalactopyranoside (IPTG) after 6 hours of growth to drive heterologous gene expression. After 48 hours of growth, $5 \mathrm{~mL}$ of ethyl-acetate containing $10 \mathrm{mg} / \mathrm{L}$ nonadecanoic acid as internal standard was added along with $500 \mu \mathrm{L}$ of $6 \mathrm{~N}$ hydrochloric acid to protonate fatty acids and increase solubility in the organic layer. The tubes were then vortexed vigorously for 45 seconds and allowed to settle. In some cases the tubes were centrifuged for $5 \mathrm{~min}$ at 4000 RPM to accelerate phase separation. The organic (top) layer was then transferred to $20 \mathrm{~mL}$ glass scintillation vials and allowed to dry overnight. Next, $500 \mu \mathrm{L}$ of a 1:2 toluene:methanol solution was added and mixed to re-solubilize fatty acids. Then $100 \mu \mathrm{L}$ of trimethylsilyldiazomethane (TMS-diazomethane, Sigma, St. Louis, MO) was added and samples capped and incubated at $37^{\circ} \mathrm{C}$ for 1 hour. Samples were then loaded directly into GC/MS vials and analyzed for fatty acid concentrations. Standard curves were made 
from pure fatty acids (Sigma) at various concentrations compared to the internal standard.

\section{Alkane analysis}

E. coli strains were pre-grown in LB media overnight, inoculated into $1 \mathrm{~mL}$ modified M9 media in glass culture tubes, and were induced with $0.5 \mathrm{mM}$ IPTG after 6 hours $\left(\mathrm{OD}_{600}\right.$ around 1.0, mid-log phase), unless mentioned specifically. To optimize alkane biosynthesis, recombinant strains were induced at different cell growth stage or with different IPTG concentration. After 48 hours, 1 $\mathrm{mL}$ ethyl acetate containing $1 \mathrm{mg} / \mathrm{L}$ octadecene as internal standard was added and the tubes were vortexed for 45 seconds. The organic (top) layer was loaded to GC/MS vials and analyzed for alkane, alcohol, and aldehyde concentration. Standard curves were made from pure chemicals (Sigma) at various concentrations compared to the internal standard.

\section{GC/MS analysis}

For all GC/MS analysis, a Shimadzu (Columbia, MD) GCMSQP2010 plus machine with an AOC-20i+s autosampler was used. Long chain alkane/alcohol/aldehyde analysis was performed using a DB-5MS column (Agilent, Santa Clara, CA) with a $0.25 \mu \mathrm{M}$ thickness, $0.25 \mathrm{~mm}$ diameter, and $30 \mathrm{~m}$ length. Splitless injection with a sampling time of 1 minute and injection temperature of $280^{\circ} \mathrm{C}$ was used and a linear velocity of $42.0 \mathrm{~cm} / \mathrm{s}$, purge flow of 2.0 $\mu \mathrm{L} / \mathrm{min}$, and column flow of $1.3 \mathrm{~mL} / \mathrm{min}$. The temperature program was as follows: $80^{\circ} \mathrm{C}$ for $5 \mathrm{~min}$ increased to $280^{\circ} \mathrm{C}$ at a rate of $20^{\circ} \mathrm{C} / \mathrm{min}$. The MS ion source temperature was $230^{\circ} \mathrm{C}$ with an interface temperature of $250^{\circ} \mathrm{C}$. A solvent cut time of 5 min was used, with an event time of $0.2 \mathrm{~s}$, scan speed of 2500 from 50-500 mass to charge $(\mathrm{m} / \mathrm{z})$ ratio. GC/MS traces of long chain alcohols and alkanes produced by recombinant $E$. coli strains were provided in Figure S3 (ESI†). Fatty acid analysis used the same GC/MS machine, but a DB-WAX column (Agilent) with a $0.25 \mu \mathrm{M}$ thickness, $0.25 \mathrm{~mm}$ diameter, and $30 \mathrm{~m}$ length. Splitless injection with a sampling time of 1 minute and injection temperature of $250^{\circ} \mathrm{C}$ and a constant pressure of $49.5 \mathrm{kPa}$, column flow of $0.95 \mathrm{~mL} / \mathrm{min}$, and purge flow of $2.0 \mathrm{~mL} / \mathrm{min}$. The temperature program was as follows: $50^{\circ} \mathrm{C}$ for $3 \mathrm{~min}$, ramped to $120^{\circ} \mathrm{C}$ at a rate of $15^{\circ} \mathrm{C} / \mathrm{min}$, then ramped to $230^{\circ} \mathrm{C}$ at a rate of $50^{\circ} \mathrm{C} / \mathrm{min}$ and held for $8 \mathrm{~min}$. The MS ion source temperature was $230^{\circ} \mathrm{C}$ with an interface temperature of $250^{\circ} \mathrm{C}$. A solvent cut time of 10 min was used, with an event time of $0.2 \mathrm{~s}$, scan speed of 1428 from 30-500 mass to charge $(\mathrm{m} / \mathrm{z})$ ratio. GC/MS traces of major fatty acids produced by engineered $E$. coli strains were provided in Figure S4 (ESI†).

Table 3. Strains and plasmids used in this study

\begin{tabular}{|c|c|c|}
\hline Name & Description & Source \\
\hline \multicolumn{3}{|l|}{ Strains } \\
\hline BL21(DE3) & $\begin{array}{l}F^{-} \text {ompT gal dcm lon hsdSB }\left(r B^{-} m B^{-}\right) \lambda(D E 3 \text { [lacI lacUV5-T7 } \\
\text { gene } 1 \text { ind1 sam7 nin5] }\end{array}$ & 38 \\
\hline BL21:AAR & BL21(DE3) with pACYC:AAR & This study \\
\hline BL21:AAR/ADO1 & BL21(DE3) with pACYC:AAR/ADO1 & This study \\
\hline BL21:AAR/ADO2 & BL21(DE3) with pACYC:AAR/ADO2 & This study \\
\hline BL21:AAR/ADO1/FAS & BL21:AAR/ADO1 + pGM44 & This study \\
\hline BL21:AAR/ADO2/FAS & BL21:AAR/ADO2 + pGM44 & This study \\
\hline BL21:AAR/ADO1/HPI & BL21:AAR/ADO1 + pRSF:HPI & This study \\
\hline BL21:AAR/ADO1/HPII & BL21:AAR/ADO1 + pRSF:HPII & This study \\
\hline BL21:AAR/ADO2/HPI & BL21:AAR/ADO2 + pRSF:HPI & This study \\
\hline BL21:AAR/ADO2/HPII & BL21:AAR/ADO2 + pRSF:HPII & This study \\
\hline BL21:AAR/ADO1/FAS/HPII & BL21:AAR/ADO1/FAS + pRSF:HPII & This study \\
\hline BL21:AAR/ADO2/FAS/HPII & BL21:AAR/ADO2/FAS + pRSF:HPII & This study \\
\hline \multicolumn{3}{|l|}{ Plasmids } \\
\hline pGM44 & $\begin{array}{l}\text { pBluescript derivative containing } F A S-B \text { and } P P T 2 \text { from } C \text {. } \\
\text { ammoniagenes }\end{array}$ & 25 \\
\hline pACYCduet & Multiple gene cloning vector & Novagen \\
\hline pRSFduet & Multiple gene cloning vector & Novagen \\
\hline pACYC:AAR & pACYCduet with Synpcc7942_1594 in MCS1 & This study \\
\hline pACYC:AAR/ADO1 & pACYC:AAR with Synpcc7942_1593 in MCS2 & This study \\
\hline pACYC:AAR/ADO2 & pACYC:AAR with Cyan7425_0398 in MCS2 & This study \\
\hline pRSF:HPI & pRSFduet with B21_03777 in MCS1 & This study \\
\hline pRSF:HPII & pRSFduet with and B21_01689 in MCS1 & This study \\
\hline
\end{tabular}

\section{Conclusions}

Overall, we demonstrate here a novel pathway for the production of long chain alcohols, aldehydes, and alkanes in $E$. coli BL21(DE3). By using a type-I actinobacterial FAS we increased flux through the PHTH-forming cyanobacterial pathway likely due to increased precursor pool concentrations.
These precursor pools are increased because the heterologous type-I FAS is not subject to feedback inhibition by acyl-ACPs. Presumably, long chain acyl-CoAs will inhibit the activity of FAS-B at a sufficient concentration, and in native actinobacterial hosts there will be regulatory mechanisms that prevent over-production of the energy dense fatty acids. Indeed, while some actionbacterial species produce mycolic acids on 
their outer leaflet, these species are not known to overproduce fatty acids. ${ }^{5,26}$ The acyl-CoA concentrations in E. coli may be maintained at a sufficiently low concentration by activity of native thioesterases or other factors to allow continuous FAS-B activity. Regardless, this pathway demonstrates a novel route to the overproduction of fatty acid derived products that has many potential applications in industrial biocatalysis.

\section{Acknowledgements}

The information, data, or work presented herein was funded in part by the Advanced Research Projects Agency-Energy (ARPA-E), U.S. Department of Energy, under Award Number DE-AR0000206. We thank Prof. Eckhart Schweizer for sharing the $F A S-B$ containing plasmid pGM44. We also would like to thank Prof. Himadri Pakrasi for generously providing the cyanobacterial genomic DNAs.

\section{Notes and references}

${ }^{a}$ Department of Chemical and Biomolecular Engineering, Institute for Genomic Biology, University of Illinois at Urbana-Champaign, Urbana, IL 61801, USA. E-mail: zhao5@illinois.edu; Phone: (217) 333-2631; Fax: (217) 333-5052

${ }^{b}$ Brookhaven National Laboratory, P.O. Box 5000, 50 Bell Avenue, Building 463, Upton, NY 11973, USA

c Departments of Chemistry, Biochemistry and Bioengineering, University of Illinois at Urbana-Champaign, Urbana, IL 61801, USA

$\dagger$ Electronic Supplementary Information (ESI) available: [Supplementary Table S1.].

1. M. R. Connor and J. C. Liao, Curr Opin Biotechnol, 2009, 20, 307315.

2. F. Zhang, S. Rodriguez and J. D. Keasling, Curr Opin Biotechnol, 2011, 22, 775-783.

3. M. Kosa and A. J. Ragauskas, Trends Biotechnol, 2011, 29, 53-61.

4. A. Schirmer, M. A. Rude, X. Li, E. Popova and S. B. del Cardayre, Science, 2010, 329, 559-562.

5. G. Gago, L. Diacovich, A. Arabolaza, S. C. Tsai and H. Gramajo, FEMS Microbiol Rev, 2011, 35, 475-497.

6. D. I. Chan and H. J. Vogel, Biochem. J., 2010, 430, 1-19.

7. E. J. Steen, Y. Kang, G. Bokinsky, Z. Hu, A. Schirmer, A. McClure, S. B. Del Cardayre and J. D. Keasling, Nature, 2010, 463, 559562.

8. F. Zhang, M. Ouellet, T. S. Batth, P. D. Adams, C. J. Petzold, A. Mukhopadhyay and J. D. Keasling, Metab Eng, 2012, 14, 653660.

9. H. Liu, C. Yu, D. Feng, T. Cheng, X. Meng, W. Liu, H. Zou and M. Xian, Microb Cell Fact, 2012, 11, 41.

10. P. Xu, Q. Gu, W. Wang, L. Wong, A. G. Bower, C. H. Collins and M. A. Koffas, Nat Commun, 2013, 4, 1409.

11. Y. J. Choi and S. Y. Lee, Nature, 2013, 502, 571-574.

12. F. Zhang, J. M. Carothers and J. D. Keasling, Nat Biotechnol, 2012, 30, 354-359.

13. J. T. Youngquist, M. H. Schumacher, J. P. Rose, T. C. Raines, M. C. Politz, M. F. Copeland and B. F. Pfleger, Metab Eng, 2013, 20, 177-186.

14. D. M. Warui, N. Li, H. Norgaard, C. Krebs, J. M. Bollinger, Jr. and S. J. Booker, J Am Chem Soc, 2011, 133, 3316-3319.

15. E. I. Lan and J. C. Liao, Metab. Eng., 2011, 13, 353-363.
16. N. Li, W. C. Chang, D. M. Warui, S. J. Booker, C. Krebs and J. M. Bollinger, Jr., Biochemistry, 2012, 51, 7908-7916.

17. J. Zhang, X. Lu and J. J. Li, Biotechnol Biofuels, 2013, 6, 86.

18. Q. Wang, X. N. Huang, J. J. Zhang, X. F. Lu, S. Y. Li and J. J. Li, Chem Commun, 2014, 50, 4299-4301.

19. Z. Rahmana, B. H. Sung, J. Y. Yi, M. Bui le, J. H. Lee and S. C. Kim, J Biotechnol, 2014, 192 Pt A, 187-191.

20. P. Jiang and J. E. Cronan, Jr., J Bacteriol, 1994, 176, 2814-2821.

21. Y. J. Lu, Y. M. Zhang and C. O. Rock, Biochem Cell Biol, 2004, 82, 145-155.

22. K. Irzik, J. van Ooyen, J. Gatgens, K. Krumbach, M. Bott and L. Eggeling, J Biotechnol, 2014, 192 Pt A, 96-101.

23. R. W. Haushalter, D. Groff, S. Deutsch, L. The, T. A. Chavkin, S. F. Brunner, L. Katz and J. D. Keasling, Metab Eng, 2015, 30, 1-6.

24. H. P. Stuible, S. Meier and E. Schweizer, Eur J Biochem, 1997, 248, 481-487.

25. H. P. Stuible, G. Meurer and E. Schweizer, Eur J Biochem, 1997, 247, 268-273.

26. C. Andre, S. W. Kim, X. H. Yu and J. Shanklin, Proc Natl Acad Sci U S A, 2013, 110, 3191-3196.

27. E. Schweizer and J. Hofmann, Microbiol Mol Biol Rev, 2004, 68, 501-517.

28. A. Kawaguchi and S. Okuda, Proc Natl Acad Sci U S A, 1977, 74, 3180-3183.

29. G. Chen, Z. Y. Peng, L. Shan, N. Xuan, G. Y. Tang, Y. Zhang, L. Li, Q. F. He and Y. P. Bi, J Biomed Biotechnol, 2012, 2012, 652579.

30. M. Harger, L. Zheng, A. Moon, C. Ager, J. H. An, C. Choe, Y. L. Lai, B. Mo, D. Zong, M. D. Smith, R. G. Egbert, J. H. Mills, D. Baker, I. S. Pultz and J. B. Siegel, ACS Synth Biol, 2013, 2, 59-62.

31. E. Jeon, S. Lee, S. O. Han, Y. J. Yoon and J. Lee, J Microbiol Biotechnol, 2012, 22, 990-999.

32. M. J. Serrano-Vega, M. Venegas-Caleron, R. Garces and E. Martinez-Force, J Chromatogr B Analyt Technol Biomed Life Sci, 2003, 786, 221-228.

33. F. Lynen, Eur J Biochem, 1980, 112, 431-442.

34. E. Schweizer and J. Hofmann, Microbiol Mol Biol Rev, 2004, 68, 501-517, table of contents.

35. E. Radmacher, L. J. Alderwick, G. S. Besra, A. K. Brown, K. J. Gibson, H. Sahm and L. Eggeling, Microbiology, 2005, 151, 2421-2427.

36. C. Dellomonaco, J. M. Clomburg, E. N. Miller and R. Gonzalez, Nature, 2011, 476, 355-359.

37. J. M. Clomburg, J. E. Vick, M. D. Blankschien, M. Rodriguez-Moya and R. Gonzalez, ACS Synth Biol, 2012, 1, 541-554.

38. F. W. Studier and B. A. Moffatt, J Mol Biol, 1986, 189, 113-130. 